\title{
A FEATURE-BASED DEFORMABLE MODEL FOR PHOTO-REALISTIC HEAD MODELLING
}

\author{
${ }^{\dagger}$ Yong-Jin Liu, ${ }^{\dagger}$ Matthew Ming-Fai Yuen, ${ }^{\ddagger}$ Shan Xiong \\ ${ }^{\dagger}$ Hong Kong University of Science and Technology, HK; ${ }^{\ddagger}$ Vanderbilt University, USA
}

Key words: Deformable model; Features; Subdivision surfaces; Facial deformation; Photogrammetry.

\begin{abstract}
We propose a feature-based approach for creating photo-realistic textured 3D head model. First, from a discrete data set of human head we generate a generic head model which is feature-based and semi-regular. Then we take a pair of photos of a human subject from two orthogonal directions. After recovering a set of $3 \mathrm{D}$ feature points on the head from photos, we build an individualized geometric model level by level. Finally we synthesize the photos into a view-independent texture map and automatically generate the texture coordinates. By mapping the texture onto the geometric model, we can efficiently generate highly photo-realistic head models for individuals.
\end{abstract}

\section{INTRODUCTION}

Pioneered by Parke [17], computer-aided modelling of human head has received considerable attentions. We can categorize these approaches into two classes based on how simple the acquisition equipment is: (1) high-cost approaches: in this category high-cost equipments are employed, such as the Cyberware color scanners $[2,15]$ and the face sculpturing robot system [12]; (2) low-cost approaches: simple low-cost equipments are needed in this category, such as the common CCD or digital cameras $[1,11,13,14,19]$.

Our approach presented in this paper falls into the category of low-cost approaches. We only use a simple digital camera to capture individual head information which is used to generate photo-realistic head models. The 
general procedure in all photo-based approaches is similar: first, the 2D features (points, lines) are extracted; then 3D geometric information is recovered from 2D features and furthermore a texture map is generated by blending taken photos; finally, by matching 2D features with predefined features on a 3D geometric model, the texture coordinates are assigned for creating a textured head model.

In our work, we observe that the format of used generic model strongly determines the efficiency of downstream operations, e.g., geometric model deformation and texture coordinates assignment. Based on model format, we briefly review the related work on low-cost approaches. For a detailed survey of this entire field, the reader is referred to the book by Parke and Waters [18] and the references therein.

\section{RELATED WORK}

Low-cost head modelling approaches can be classified according to the format in which the head models are represented. There are two popular formats widely used for head models: parametric surfaces and polygonal surfaces.

Parametric surfaces describe head models mathematically in parametric equations. Due to the intrinsic parameterisation, it is straightforward to deform the surface and generate texture coordinates. However, it is difficult to model a highly detailed human head using parametric surface, since either the number of control points is increased rapidly, or a network of parametric patches need to be constructed. A typical work using parametric surface for head modelling is presented in [5].

Compared with parametric surfaces, polygonal surfaces are more flexible in modelling fine details. Most of existing low-cost approaches use irregular polygonal mesh for head modelling. However, after specifying a set of feature vertices on a generic model, the applications of polygonal models are confronted by the difficulties of specifications for remaining vertices. Addressing this problem, a number of techniques are proposed: Kurihara and Arai [13] project the vertices into cylindrical parameter plane and use Delaunay triangulation of feature vertices; Ip and Yin [11] look for $N$ nearest feature vertices around each non-feature vertex; Pighin et al. [19] and Akimoto et al. [1] build a scatter data interpolation function; Lee and Thalmann [14] use a Dirichlet free-form deformation.

With the development of multiresolution techniques, subdivision surfaces have been widely studied [21]. Recent works [8, 10, 22] have extended subdivision surfaces to arbitrary topology and therefore offer a bridge between parametric surfaces and polygonal surfaces. In our work, we use a 
multi-level displaced subdivision surface for head modelling, which shows advantages in key parts of photo-realistic head modelling, i.e., local and smooth deformation, detail presentation and texture coordinates assignment.

\section{FEATURE-BASED SEMI-REGULAR GENERIC HEAD MODEL GENERATION}

In our approach, we use a displaced butterfly subdivision scheme to build our generic head model from a discrete data set of a human head.

\subsection{Background on Butterfly Subdivision Surface}

We follow the notation in Hoppe [10] to describe polygonal model: a mesh $M$ is a pair $(K, V)$, where $K$ is a simplicial complex specifying the connectivity of the vertices, edges, and faces; $V=\left\{\mathbf{v}_{1}, \mathbf{v}_{2}, \cdots\right\}, \mathbf{v}_{i} \in \mathbf{R}^{3}$ is a set of vertex position defining the shape of $M$ in $\mathbf{R}^{3}$.

The butterfly scheme $S$ is an interpolating subdivision scheme for triangle meshes. Given $S$, one subdivision step carries a mesh $M^{i}=\left(K^{i}, V^{i}\right)$ to a mesh $M^{i+1}=\left(K^{i+1}, V^{i+1}\right)$ by $M^{i+1}=S M^{i}$. The set $V^{i+1}$ can be classified into subsets $V_{v}^{i+1}$ and $V_{e}^{i+1}$, where $V_{v}^{i+1}=V^{i}$ is related to the vertices in $M^{i}$ and $V_{e}^{i+1}$ is related to edges in $M^{\prime}$. The subdivision surface is then defined by recursively applying the refinement $M^{i+1}=S M^{i}$ on an initial control mesh $M^{0}$. Butterfly scheme is first introduced by Dyn [6,7], which generates $C^{1}$-continuous limit surface on a regular control mesh. Zorin [22] proposes a modified butterfly scheme for the generation of $C^{1}$-continuous surfaces of arbitrary topology. Since $V_{v}^{i+1}=V^{i}$, we only calculate $V_{e}^{i+1}$ from $V^{i}$. Although we do not iteratively subdivide $M^{0}$ infinitely, for each vertex in $V^{i}, i=0,1,2, \cdots$, its exact tangent plane on the limit surface, spanned by two orthogonal tangent vectors $t_{1}$ and $t_{2}$, can be computed by analysing the eigen-structure of the local subdivision matrix $\mathbf{S}_{n}[8,21,22]$.

\subsection{Discrete Data Preprocessing}

Given a discrete data set of a human head $H=\left\{\mathbf{h}_{1}, \mathbf{h}_{2}, \cdots, \mathbf{h}_{n}\right\}$ (cf. Fig. 1), we extract its topological information by looking for an implicit function $f$, such that $f\left(\mathbf{h}_{i}\right)=0, \forall \mathbf{h}_{i} \in H$. We numerically determine the implicit function $f$ based on the sign distance function in [9], as summarized in the following three steps.

1. Build a Riemannian graph $G=(V, E)$ to estimate normal direction for each data point. 

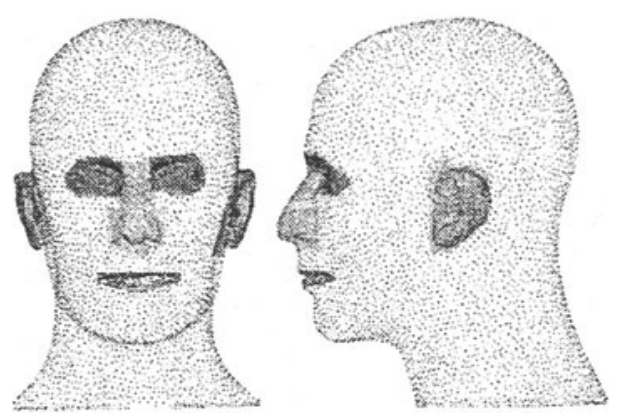

Figure 1. A discrete data set of a human head
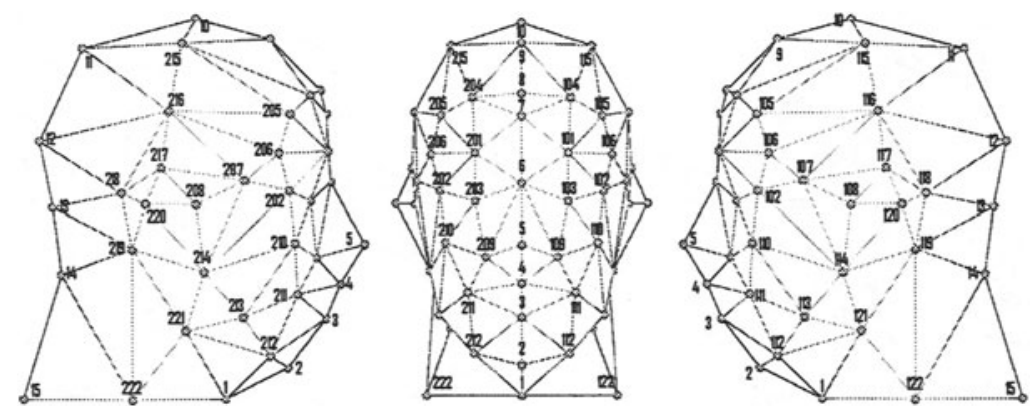

Figure 2. The structure of the predefined 3D feature mesh

2. Extract the minimum spanning tree for graph $G$, which is further traversed in a depth-first search to orient the normal vectors.

3. Use a $k$-nearest-neighbour searching algorithm to numerically determine the value $f(\mathbf{p})$ and the gradient $\nabla f(\mathbf{p})$ for an arbitrary point $\mathbf{p} \in \mathbf{R}^{3}$.

\subsection{Feature Definition}

On the human head we define a set of feature points and organize them into a triangular mesh, which we refer to as a feature mesh $M^{0}=\left(K^{0}, V^{0}\right)$. Fig.2 illustrates the structure of $K^{0}$. The geometric positions of feature points $V^{0}$ in $\mathbf{R}^{3}$ are specified by corresponding points in discrete data. The rules we used to define the $\mathrm{ID}$ of feature points are as follows:

- The feature point whose ID number is smaller than 100 lies in symmetry plane.

- The feature point whose ID number is larger than 100 and smaller than 200 lies in left face. 


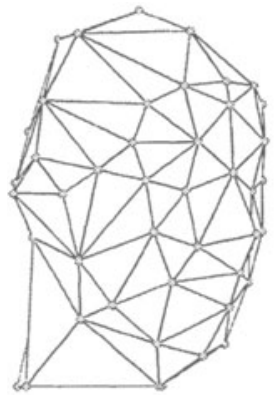

(a) $M^{0}$

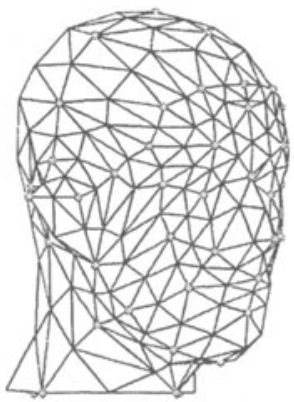

(b) $M^{I}=\left(M^{0}, D^{I}\right)$

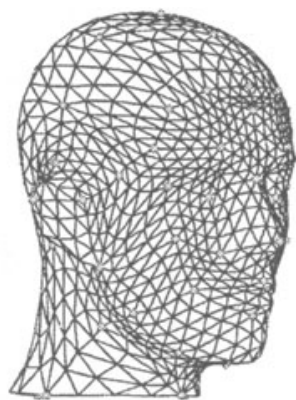

(c) $M^{2}=\left(M^{1}, D^{2}\right)$

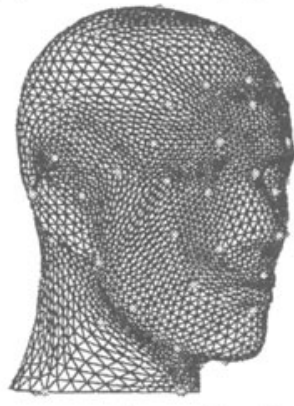

(d) $M^{3}=\left(M^{2}, D^{3}\right)$

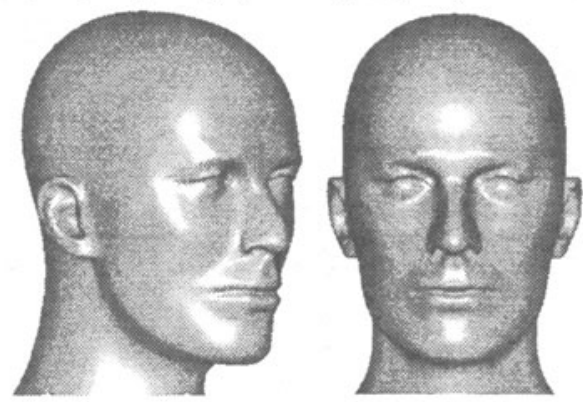

(e) $M^{4}=\left(M^{3}, D^{4}\right)$

Figure 3. Mesh refinement and its multi-level representation

- The feature point whose ID number is larger than 200 lies in right face, and the feature point $2 \mathrm{ij}$ is the symmetrical point of $1 \mathrm{ij}$ according to symmetry plane.

\subsection{Mesh Refinement}

Given a starting mesh $M^{0}$, we use a displaced butterfly scheme to refine $M^{0}$ to capture full details of the data $H$. For a given mesh $M^{i}$, one refinement step from $M^{i}=\left(K^{i}, V^{i}\right)$ to $M^{i+1}=\left(K^{i+1}, V^{i+1}\right)$ consists of two substeps: a subdivision step and a displacing step. First, the subdivision step refines mesh $M^{i}=\left(K^{i}, V^{i}\right)$ to an intermediate mesh $M^{i+1}=\left(K^{i+1}, V^{i+1}\right)$ using the modified butterfly scheme [22]. For each vertex in $V_{e}^{i+1}$, its geometric position and two orthogonal tangent vectors $t_{1}$ and $t_{2}$ on the limit surface can be calculated using edge and tangent masks. Then in the displacing step $M^{i+1}=\left(K^{i+1}, V^{i+1}\right) \rightarrow M^{i+1}=\left(K^{i+1}, V^{i+1}\right)$, we establish a local frame $\mathbf{F}^{i+1}(j)$ for each vertex $\hat{v}_{j}^{i+1} \subset V_{e}^{i+1} . \mathbf{F}^{i+1}(j)$ is built up as $\mathbf{F}^{i+1}(j)=\left(t_{1}^{i+1}(j), t_{2}^{i+1}(j), n^{i+1}(j)\right)$, where $n^{i+1}(j)=t_{1}^{i+1}(j) \times t_{2}^{i+1}(j), t_{1}^{i+1}(j)$ and $t_{2}^{i+1}(j)$ are two unit orthogonal tangent vectors at the position of $\widehat{v}_{j}^{i+1}$. Along the normal direction $n^{i+1}(j)$ in $\mathbf{F}^{i+1}(j)$, we offset each vertex $\hat{v}_{j}^{i+1}$ to a new position $v_{j}^{i+1}$, where $f\left(v_{j}^{i+1}\right)=0$. 
Starting from $M^{0}$, we iteratively perform refinement until we reach the final level 4. As illustrated in Fig. 3, our mesh refinement operation produces a mesh hierarchy $M^{0}, M^{1}, M^{2}, M^{3}, M^{4}$, i.e., our generic head model is semi-regular and is feature-based.

\subsection{Multi-Level Displaced Mesh Representation}

We represent our feature-based semi-regular head model in multi-levels. Recall that in one refinement step, we first subdivide a mesh $M^{i}=\left(K^{i}, V^{i}\right)$ to an intermediate mesh $M^{i+1}=\left(K^{i+1}, V^{i+1}\right)$ using a modified butterfly scheme $S$, i.e., $\bar{M}^{i+1}=S M^{i} . \forall \hat{v}_{j}^{i+1} \subset V_{e}^{i+1}, \quad \hat{v}_{J}^{i+1}=\mathbf{S}_{n} V_{\text {mask }}^{i}$, where $\mathbf{S}_{n}$ is the local subdivision matrix of the scheme $S$, and $V_{\text {mask }}^{\prime} \subset V^{\prime}$ is a local vertex set in the edge mask of $\hat{v}_{j}^{i+1}$. Then in the displacing step, we offset each vertex $\hat{v}_{j}^{i+1}$ to $v_{j}^{i+1}$ along its normal direction $n^{i+1}(j)$ with magnitude $a_{j}^{\cdots}$, i.e.,

$$
v_{j}^{i+1}=\hat{v}_{j}^{i+1}+d_{j}^{i+1} n^{i+1}(j)=\mathrm{S}_{n} V_{\text {mask }}^{i}+d_{j}^{i+1} n^{i+1}(j)
$$

Since local frames $\mathbf{F}^{i+1}(j)$ are self-determined by $S$ and $V^{i}$, the vertex set $V^{i+1}$ is fully determined by $\left(S, V^{i}, D^{i+1}\right)$, where $D^{i+1}$ is a detail set $D^{i+1}$ that consists of scalars $d_{j}^{i+1}$ for each vertex $v_{j}^{i+1} \subset V_{e}^{i+1}$, and the topology $K^{i+1}$ is fully determined by $\left(S, K^{i}\right)$. Therefore, our mesh hierarchy can be represented by a feature mesh together with a multi-level scalar detail set, i.e.,

$$
M^{4}=\left(M^{3}, D^{4}\right)=\left(M^{2}, D^{3}, D^{4}\right)=\left(M^{1}, D^{2}, D^{3}, D^{4}\right)=\left(M^{0}, D^{1}, D^{2}, D^{3}, D^{4}\right)
$$

\section{PHOTO-REALISTIC HEAD MODELING}

We take a pair of photos of a human subject from the front and side views. We flip the side view photo to obtain one more side view photo. Then we determine a set of 2D features on the photos as follows.

\subsection{D Feature Determination}

We organize the vertices in $M^{0}$ into seven feature lines. Each feature line has its vision characteristics on photos: the projection of each feature line should be viewable in one or more photos. For each feature line we define several key feature points in it and different feature lines may share common feature points. We first project each feature line onto its viewable photos and interactively determine the corresponding 2D key feature points. We then use the structure snake algorithm [14] to determine the remaining feature point. One example is illustrated in Fig. 4. 

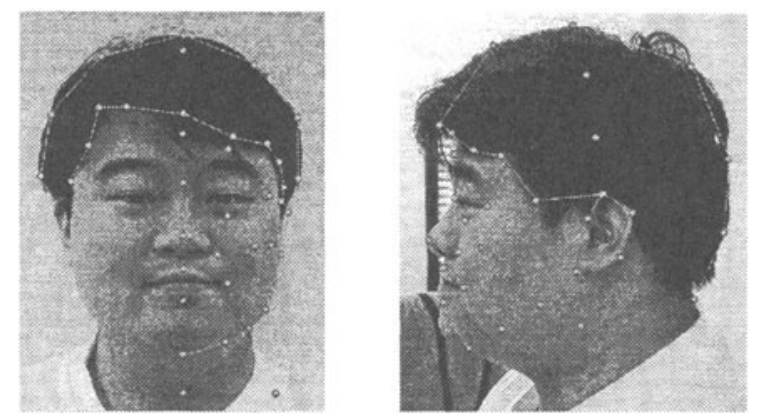

Figure 4. 2D feature determination for individual

To allow more flexibility, we relax the requirement of taking photos from strictly orthogonal directions. We associate a rotation matrix $\boldsymbol{R}$ and a translation vector $\boldsymbol{T}$ to each photo. Using the feature points which are viewable simultaneously on two photos, we calculate $\boldsymbol{R}$ and $\boldsymbol{T}$ for each photo using the structure-from-motion algorithm [20], which is first solved using the Levenberg-Marquardt algorithm [20] and later simplified to a set of linear equations [19].

With $\boldsymbol{R}$ and $\boldsymbol{T}$ for each photo, we recover the 3D coordinates of 2D feature points: if the feature point is viewable in two photos, its exact 3D position can be calculated; if the feature point can only be viewed in one photo, we find its $3 \mathrm{D}$ position in the viewing ray nearest to the corresponding vertex in generic model.

\subsection{Model Deformation for Individuals}

After we specify the $3 \mathrm{D}$ position for each vertex in $M^{0}$ for individuals, we deform the generic model into a lifelike individualized model using the multi-level representation $\left(M^{0}, D^{1}, D^{2}, D^{3}, D^{4}\right)$.

Given an individualized feature mesh $\bar{M}^{0}$, we add back the detail part $\left(D^{1}, D^{2}, D^{3}, D^{4}\right)$ to synthesize an individualized model $\bar{M}^{4}$. Note that in generic model generation, each scalar detail coefficient $d_{j}^{i}$ offsets the vertex $v_{j}^{i}$ along the normal direction $n^{i}(j)$. Starting from $\bar{M}^{0}$, we first use the same butterfly scheme $S$ to refine a mesh $\bar{M}^{i}$ to an intermediate mesh $\overline{\bar{M}}^{i+1}=S \bar{M}^{i}$. We then compute a local frame $\mathbf{F}^{i+1}(j)=\left(\bar{t}_{1}^{i+1}(j), \bar{t}_{2}^{i+1}(j), \bar{n}^{i+1}(j)\right)$ on the fly for each new generated vertex $\overline{\bar{v}}_{j}^{i+1} \subset \bar{V}_{i}^{i+1}$ based on $\overline{\bar{M}}^{i}$ and $S$. Then along the normal direction $\bar{n}^{i+1}(j)$, we offset each vertex $\bar{v}_{j}^{i+1}$ with a magnitude $d_{j}^{i+1}$. Similar to equation (1), in a mathematic form,

$$
\bar{v}_{j}^{i+1}=\mathrm{S}_{n} V_{\text {mask }}^{i}+d_{j}^{i+1} \bar{n}^{i+1}(j)
$$

Now our mesh synthesis process can be described as

$$
M^{i}=\left(K^{i}, V^{i}\right) \Rightarrow M^{i+1}=\left(K^{i+1}, V^{i+1}\right) \text {, }
$$

where $K^{i+1}=S K^{i}$ and $\bar{V}^{i+1}$ is determined by $\left(S, V^{i}, D^{i+1}\right)$ using equation (3). 


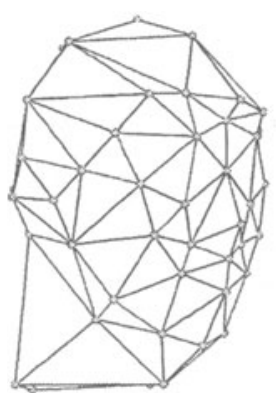

(a) $\bar{M}^{\circ}$

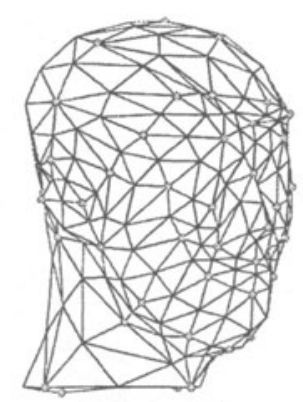

(b) $\bar{M}^{1}=\left(\bar{M}^{0}, D^{1}\right)$

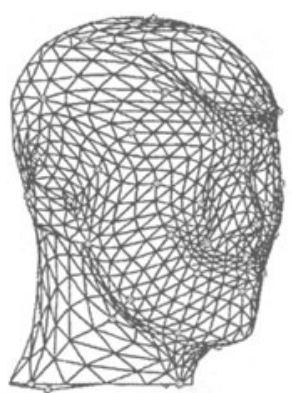

(c) $\bar{M}^{2}=\left(\bar{M}^{1}, D^{2}\right)$

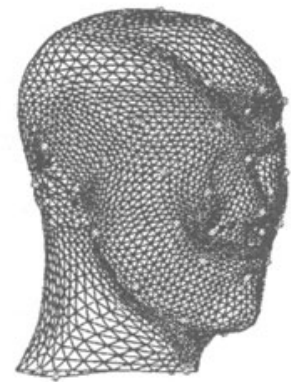

(d) $\bar{M}^{3}=\left(\bar{M}^{2}, D^{3}\right)$
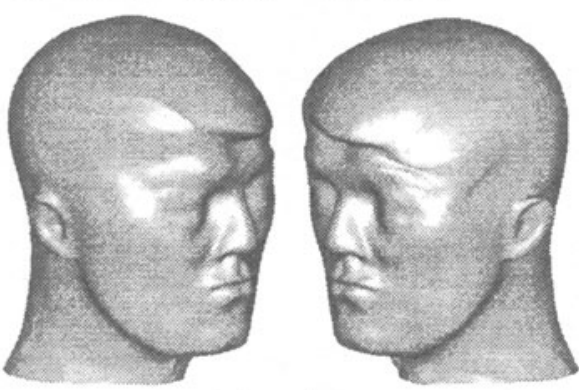

(e) $\bar{M}^{4}=\left(\bar{M}^{3}, D^{4}\right)$

Figure 5. Individualized mesh generation level by level

We synthesize the individualized model level by level based on $S$ and $\left(D^{1}, D^{2}, D^{3}, D^{4}\right)$. See Fig. 5 for an illustration. Since for each level $i$, every new generated vertex $\bar{v}_{j}^{i}$ is offset in its own local frame $F^{i}(j)$ that build upon a $C^{1}$-continuous limit surface, our deformation scheme efficiently guarantees the properties of locality and smoothness.

\subsection{Texture Extraction and Texture Coordinates Generation}

Generally, there are two types of texture maps used in photo-realistic head modelling: view-dependent and view-independent texture maps. Viewdependent texture map blends photos dynamically according to the current viewpoint, and thus, high-frequency details are visible $[11,19]$. The price paid for dynamically blending is the need of high memory requirement and low response-speed. Compared with view-dependent maps, the viewindependent texture map blends all photos together into a single texture map, and thus, can support rapid display of textured head model from any viewpoints $[1,13,14]$. However, due to the fixed blending weights, the result of view-independent mapping is slightly blurred. 


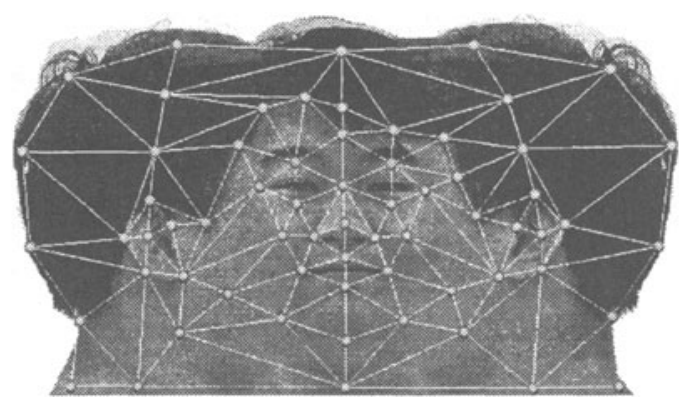

Figure 6. View-independent texture map with the 2D feature mesh

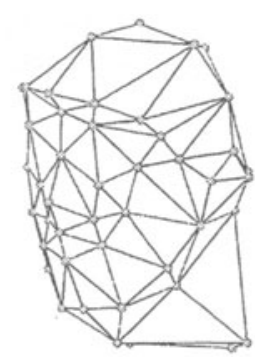

(a) $\bar{M}^{0}$

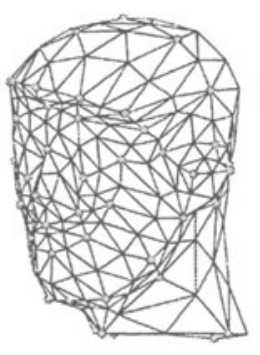

(b) $\bar{M}^{1}$

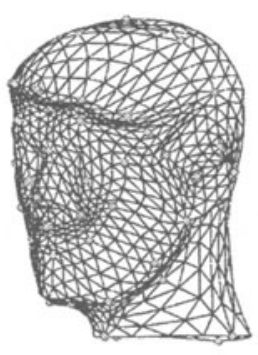

(c) $\bar{M}^{2}$

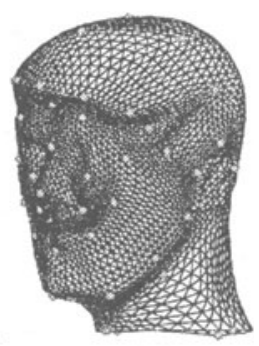

(d) $\bar{M}^{3}$
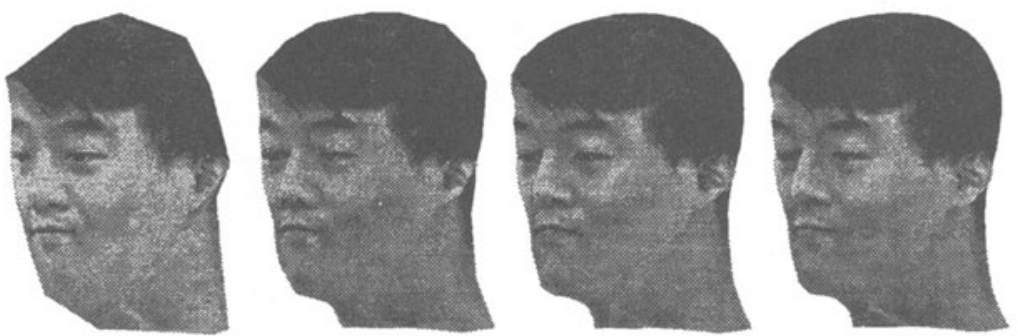

(e) $\bar{M}^{0}+\bar{M}_{2 D}^{0}$

(f) $\bar{M}^{1}+\bar{M}_{2 D}^{1}$

(g) $\bar{M}^{2}+\bar{M}_{2 D}^{2}$

(h) $\bar{M}^{3}+\bar{M}_{2 D}^{3}$
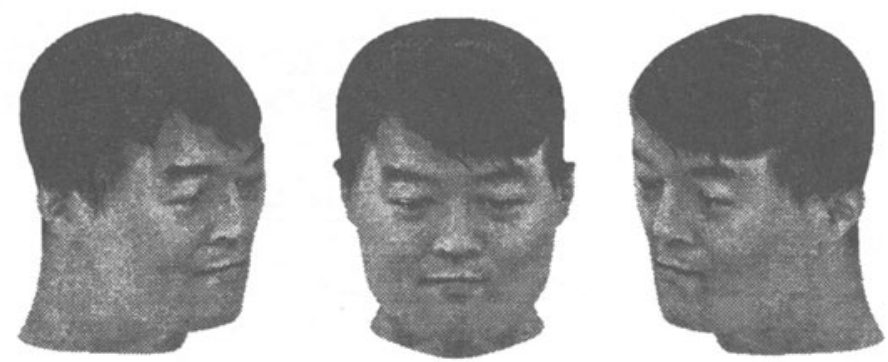

(i) $\bar{M}^{4}+\bar{M}_{2 D}^{4}$

Figure 7. Multi-level texture mapping with automatic texture coordinate generation 
In our work, we use a view-independent texture map. Based on the set of 2D feature points on each photo, we blend these photos into a viewindependent texture map (cf. Fig. 6), which is used for rendering textured head model from any viewpoints. We follow the process in Lee and Thalmann [14] to extract the texture map: photos are first distorted with a predefined index of feature lines, and then, sewed together along feature lines; subsequently a multi-resolution spline technique [3] is applied to remove the boundaries around the sewing regions.

Benefited from our feature-based semi-regular head model, we can automatically assign texture coordinates for the vertices in individualized model $\bar{M}^{4}$. On the resulting texture map, we build a 2D feature mesh $M_{2 D}^{0}$, whose vertices are determined by 2D feature points on individual photos. $M_{2 D}^{0}$ is served as the 2D development of 3D feature mesh $\bar{M}^{0}$ by splitting it along the feature line 10-11-12-13-14-15 (cf. Fig. 2). We then apply the same butterfly scheme on the 2D feature mesh, i.e., $M_{2 D}^{i+1}=S M_{2 D}^{i}$. Thanks to the semi-regular structure of our head model, except the splitting feature line, there is a one-to-one correspondence between the 2D and 3D feature meshes and so hold between the refined meshes $M_{2 D}^{0}, M_{2 D}^{1}, M_{2 D}^{2}, M_{2 D}^{3}, M_{2 D}^{4}$ and $M^{0}, M^{1}, M^{2}, M^{3}, M^{4}$. Note that for $\bar{M}^{i}$ on each level $i$, every vertex along the splitting feature line 10-11-12-13-14-15 has two separate texture coordinates but identical positions. The 2D meshes $M_{2 D}^{i}$ turned out to be the texture meshes of 3D head models $\bar{M}^{\prime}$. To generate a lifelike textured model for individuals, we simply map $M_{2 D}^{i}$ to $\bar{M}^{\prime}$ level by level (cf. Fig. 7).

\section{CONCLUSION AND FUTURE WORK}

In this paper, we propose a feature-based deformable model for photorealistic head modelling. The key part of our approach is the multi-level displaced representation of our deformable head model. We have demonstrated that using our presented technique, we can generate highly photo-realistic head model for individuals fast and efficiently.

Our feature-based deformable model can be further used in a wide range of applications, which include facial animation, model-based tracking from video, scalar compression for geometry and use of detailed textures for displacements.

\section{REFERENCES}

1. Akimoto T., Suenaga Y. Automatic creation of 3D facial models. IEEE Computer Graphics \& Applications 1993; 13(5):16-22. 
2. Blanz V., Vetter T. A morphable model for the synthesis of 3D faces. Proceedings of SIGGRAPH '99, ACM SIGGRAPH, New York 1999; pp.187-94.

3. Burt P.J., Adelson E.H. A multiresolution spline with application to image mosaics. ACM Transactions on Graphics, 1983; Vol. 2, No. 4, pp 217-36.

4. Catmull E., Clark J. Recursively generated B-spline surfaces on arbitrary topological meshes. Computer-Aided Design 1978; 10(6):350-355.

5. DeCarlo D., Mataxas D., Stone M. An anthropometric face model using variational techniques. Proceedings of SIGGRAPH '98, ACM SIGGRAPH, 1998; pp 67-74.

6. Dyn N., Levin D., Gregory J.A. A 4-point interpolatory subdivision scheme for curve design. Computer Aided Geometric Design 1987; 4:257-68.

7. Dyn N., Levin D., Gregory J.A. A butterfly subdivision scheme for surface interpolation with tension control. ACM Transaction on Graphics 1990; 9(2):160-69.

8. Halstead M. Kass M., DeRose T. Efficient, fair interpolation using catmull-clark surfaces. Proceedings of SIGGRAPH '93, ACM SIGGRAPH, Addison Wesley 1993; pp 35-43.

9. Hoppe H., DeRose T., Duchamp T., Mcdonald J., Stuetzle W. Surface reconstruction from unorganized points. Proceedings of SIGGRAPH '92, ACM SIGGRAPH, Addison Wesley 1992; pp 71-78.

10. Hoppe H., DeRose T., Duchamp T., Halstead M., Jin H., McDonald J., Schweitzer J., Stuetzle W. Piecewise smooth surface reconstruction. Proceedings of SIGGRAPH '94, ACM SIGGRAPH, Addison Wesley 1994; pp 295-302.

11. Horace H.S.I., Yin L.J. Constructing a 3D individualized head model from two orthogonal views. Visual Computer, 1996; vol.12, no.5, pp.254-66.

12. Ko H., Kim M.S., Park H.G., Kim S.W. Face sculpturing robot with recognition capability. Computer Aided Design 1994; 26(11):814-21.

13. Kurihara T., Arai K. A transformation method for modeling and animation of the human face from photographs. Proceeding of Computer Animation '91, Springer-Verlag Tokyo 1991; pp. 45-58.

14. Lee W.S., Magnenat-Thalmann N. Fast head modeling for animation. Image \& Vision Computing 2000; 18(4):355-64.

15. Lee Y., Terzopoulos D., Waters K. Realistic modeling for facial animation. Proceedings for SIGGRAPH '95, New York 1995; pp 55-62.

16. Loop C. Smooth subdivision surfaces based on triangles. Master thesis, 1987, Department of Mathematics, University of Utah.

17. Parke F.I. A parametric model for human faces. PhD dissertation, 1974, Univ. Utah.

18. Parke F.I., Waters K. Computer facial animation. Wellesley, Massachusetts, 1996.

19. Pingin F., Hecker J., Lischinski D., Szeliski R., Salesin D.H. Synthesizing realistic facial expressions from photographs. Proceedings of SIGGRAPH '98, ACM SIGGRAPH, Addison Wesley 1998; pp 75-84.

20. Szeliski R., Kang S.B. Recovering 3D shape and motion from image streams using nonlinear least squares. Journal of Visual Communication and Image Representation, 1994; 5(1):10-28.

21. Zorin D., Schroder P. Subdivsion for modeling and animation. SIGGRAPH Course Notes, 2000.

22. Zorin D., Schroder P., Sweldens W. Interpolating subdivision for meshes with arbitrary topology. Proceedings of SIGGRAPH '96, ACM SIGGRAPH, Addison Wesley 1996; pp 189-92. 